\title{
Saccade dynamics in peripheral vs central sixth nerve palsies
}

\author{
Agnes M.F. Wong, MD, PhD, FRCSC; Kylen McReelis, MD, FRCSC; and James A. Sharpe, MD, FRCPC
}

\begin{abstract}
Objective: To investigate differences between peripheral idiopathic and central sixth nerve palsies from brainstem damage by comparing peak velocities and durations of horizontal saccades. Methods: Fourteen patients with unilateral incomplete sixth nerve palsies caused by idiopathic, presumed ischemic, peripheral damage, 5 with incomplete central (fascicular) palsy caused by brainstem lesions, and 10 controls were studied. Palsies under 1 month in duration were designated as acute and those of longer duration were chronic. Among peripheral palsies, five were acute, nine were chronic. Among central palsies, two were acute, three were chronic. Subjects made \pm 10 deg horizontal saccades while wearing search coils. Serial recordings were made in seven patients with acute palsy (five peripheral, two central). Results: Centrifugal abducting saccadic velocities in the paretic eye were subnormal in both central and peripheral acute palsies, as anticipated from lateral rectus weakness. In chronic central palsies, abducting velocities in the paretic eye remained reduced. However, in chronic peripheral palsies, velocities became normal in the tested range of excursion, within 2 months of onset, despite persisting abduction deficit. Conclusions: Saccade peak velocities are reduced and their durations are prolonged in the field of action of acutely palsied peripheral and central nerves. Speeds remain reduced in chronic central (fascicular) palsies, consistent with limited regeneration within the brain. Saccade speeds are repaired in chronic peripheral palsies, probably by remyelination and axonal regeneration, and perhaps also by central monocular adaptation of innervation selectively to the paretic eye, in order to drive both eyes rapidly and simultaneously into the paretic field of motion.
\end{abstract}

NEUROLOGY 2006;66:1390-1398

The study of abducens nerve palsy provides a unique opportunity to investigate differences in repair properties between the central and peripheral nervous systems, since the nerve has a substantial course both within and outside the brain, where it is vulnerable to damage. In addition, the abducens nerve only innervates one muscle, the lateral rectus, so its function can be readily quantified by assessing the dynamic properties of that muscle alone.

Clinical testing of paralytic strabismus emphasizes examination of static deviations of the eyes. Information on saccade performance after sixth nerve palsy is sparse. Abducens nerve palsy lowers saccade amplitude and velocity in the direction of action of the paretic lateral rectus muscle. ${ }^{1-6}$ The effects of duration (acute vs chronic) and location (peripheral vs central) of abducens nerve damage on saccade dynamics had not been systematically investigated, nor had the potential of recovery been previously addressed.

Additional material related to this article can be found on the Neurology Web site. Go to www.neurology.org and scroll down the Table of Contents for the May 9 issue to find the title link for this article.
In this study, we investigated patients with unilateral sixth nerve palsy and assessed the saccades of both the paretic and non-paretic eyes during monocular viewing with each eye. We studied the effects of peripheral vs central (fascicular) sites of damage and duration of the palsy on speed of saccades. The results indicate that saccadic speed differs between acute and chronic palsies, and between central and peripheral damage to the nerve. Preliminary results have been published as an extended abstract. ${ }^{7}$

Methods. Clinical assessment and imaging. Nineteen patients with unilateral peripheral sixth nerve palsy were recruited from the Neuro-ophthalmology Unit at the University Health Network. Complete histories and detailed ophthalmic and neurologic examinations were performed, recording the duration and age at onset of diplopia, the presence or absence of risk factors for ischemia (diabetes mellitus and hypertension), and associated neurologic symptoms and signs. We selected only patients with sixth nerve palsies that did not recover within 1 month of onset of diplopia and had clinically evident limitation of abduction. Patients with diplopia of less than 1 month duration were classified as having acute palsy; all others were designated here as chronic. The magnitude of strabismus was measured using the prism and cover test. Ranges of duction were estimated independently by two examiners (A.W. and J.A.S.) who graded the abduction defect as the estimated percentage of the normal abduction in the other eye.

From the Department of Ophthalmology and Vision Sciences (A.M.F.W., K.M., J.A.S.) and Division of Neurology (A.M.F.W., J.A.S.), University Health Network, University of Toronto, Ontario, Canada.

Supported by the New Investigator Award (MSH 55058) (A.M.F.W.) and grants MT 5404, ME 5509 (J.A.S.), and MOP 57853 (A.M.F.W. and J.A.S.) from the Canadian Institutes of Health Research (CIHR), and the University Health Network Ophthalmology (A.M.F.W.) and Neurology (J.A.S.) Practice Plans.

Disclosure: The authors report no conflicts of interest.

Presented in part at the 29th Annual North American Neuro-ophthalmology Society (NANOS) Meeting; Salt Lake City, UT; February 9-13, 2003.

Received September 28, 2005. Accepted in final form January 23, 2006.

Address correspondence and reprint requests to Dr. Agnes Wong, Elm Wing S102D, The Hospital for Sick Children, 555 University Avenue, Toronto, Ontario, Canada M5G 1X8; e-mail: agnes.wong@utoronto.ca

1390 Copyright (0) 2006 by AAN Enterprises, Inc. 
Appropriate tests were performed, when indicated, to rule out myasthenia gravis, thyroid ophthalmopathy, and other orbital diseases, and to detect intracranial lesions.

Serial axial and sagittal T1- and T2-weighted MR images with gadolinium enhancement were obtained (slice thickness $=5 \mathrm{~mm}$ ) for all patients under 50 years of age and those with other neurologic signs. In this investigation, CT images of the head with contrast were obtained in all patients with ischemic risk factors and for patients over 50 years of age, although imaging is not our standard practice for such patients unless the palsy persists after several weeks. If CT imaging was normal, patients were followed up after about 3 months. Those without improvement of their palsy at 3 months and those with an abnormal CT scan were further investigated with MR imaging.

Ten normal subjects served as controls ( 5 men; mean age $=$ $49 \pm 12$ years; median age $=55$ years; age range $=19$ to 69 years). The research protocol followed the tenets of the Declaration of Helsinki and the Ethics Review Board of the University Health Network and informed consent was obtained from each participant.

Eye movement recordings. Visual stimuli and experimental protocol. Participants fixated a red laser spot of $0.25^{\circ}$ in diameter, rear-projected onto a vertical flat screen $1 \mathrm{~m}$ away from the nasion. Starting in the center position (at eye level in patient's midsagittal plane), the laser stepped to $10^{\circ}$ right, then back to center, then $10^{\circ}$ left, cycling through this pattern 20 times. Head position was stabilized with an occipital support and monitored by a search coil taped to the forehead. With one eye covered, patients were instructed to follow the laser spot as it stepped among positions. At each position the laser halted for 3 seconds. The target sequences were then repeated with the other eye covered. To avoid fatigue, breaks for 1 to 3 minutes were provided approximately every 2 minutes.

Recordings of eye movement and calibration. Eye positions were measured by a three-dimensional magnetic search coil technique, using $6 \mathrm{ft}(183 \mathrm{~cm})$ diameter coils field arranged in a cube (CNC Engineering, Seattle, WA). In each eye, the patient wore a dual-lead scleral coil annulus designed to detect horizontal, vertical, and torsional gaze positions (Skalar Instrumentation, Delft, Netherlands). The coils were calibrated on a protractor before insertion on the eye. Calibration was verified by fixation with each eye independently (with the fellow eye occluded), which was possible because saccades were tested within the range of spared duction of the paretic eye. Horizontal eye movements were tested with saccades to steps of the laser target. Any coil slippage was assessed by monitoring offsets in eye position signal during testing. Consistency of calibrated positions after each eye movement provided evidence that the coil did not slip on the eye. The linear range for horizontal movement was over 30 degrees. Crosstalk between dimensions was less than one degree and noise was less than 0.1 degrees. Positions were calculated using rotation vectors. Position data were anti-alias filtered using four pole low-pass Butterworth filters with a corner frequency $(-3 \mathrm{db})$ of $90 \mathrm{~Hz}$ and then digitized at $500 \mathrm{~Hz}$. Data were recorded on disc for off-line analysis.

Saccade onsets and ends were detected with a $30 \mathrm{deg} / \mathrm{second}$ threshold, which excludes postsaccadic drifts. We used a digital five-point Savitsky-Golay differentiator ${ }^{8}$ that does not attenuate until above $75 \mathrm{~Hz}$ to compute peak velocity. For each eye movement with a velocity greater than $30 \mathrm{deg} / \mathrm{sec}$, peak velocity was marked. For each selected peak velocity, the time when eye velocity surpassed or dropped below 5\% of maximum velocity was taken as the beginning and the end of a saccade. Cursors then marked the beginning and end of saccades in the corresponding eye position channel.

Data analyses. Based on clinical examinations and imaging findings, patients were classified into two groups: those with idiopathic, presumed ischemic, peripheral palsy and those with central fascicular palsy. Patient characteristics, including age and abduction deficit (acute vs chronic), were compared using $t$ tests. For nonparametric data (duration of palsy), Wilcoxon rank-sum tests were used.

Saccade amplitudes, peak velocities, and durations were analyzed for the paretic and non-paretic eyes. In all patients, the peak velocity-amplitude and duration-amplitude relationships in both the paretic and non-paretic eyes did not differ between viewing with the paretic or the non-paretic eye. Here we report only the peak velocity-amplitude and duration-amplitude relationships during paretic eye viewing; the relationships during non-paretic eye viewing were similar. Data from patients with peripheral or central palsy were analyzed by a 3 (between) x 4 (within) nested repeated measures analysis of variance (ANOVA). The betweensubjects factor was the stages of palsy (acute, chronic, and controls) and the within-subjects factor was the amplitude of saccades in bins ( 4 to 6,6 to 8,8 to 10 , and 10 to $12 \mathrm{deg}$ ). For patients who had serial recordings in both the acute and chronic stages, the effects of the stages of palsy were analyzed by a crossed design repeated measures ANOVA. The specific effects of each factor were analyzed further with post-hoc Sidak tests, with significance set at $p<0.05$ level.

Results. General characteristics of patients. Nineteen patients (12 men, 7 women) were recruited. Fourteen of the 19 patients had idiopathic, presumed microvascular ischemic, ${ }^{9}$ peripheral palsy (table 1 ). Nine of these 14 patients were men. All 14 peripheral cases had brain and sixth nerve imaging; 10 patients had normal MR imaging and 4 had normal CT scanning (see table 1). Some "idiopathic" sixth nerve palsies are associated with brainstem infarction, ${ }^{10}$ but despite imaging, we did not identify any such case. Four patients had identifiable risk factors for ischemia, namely hypertension or diabetes mellitus. Five patients were tested within 1 month of symptom onset. Serial eye movement recordings were performed on these five patients with acute peripheral palsy at presentation and at 2 months after symptom onset. The mean duration of diplopia (at the time of eye movement recording) in acute peripheral palsies ( 5 patients) was 3 weeks, whereas the mean duration in chronic peripheral palsies (14 patients) was $27 \pm 24$ months. The mean age in acute peripheral palsies $(n=5)$ was $52 \pm 18$ years (median age $=56$ years; age range $=30$ to 75 years), whereas the mean age in chronic peripheral palsies $(\mathrm{n}=14)$ was $63 \pm 10$ years (median age $=64$ years; age range $=46$ to 77 years).

Five patients had central (fascicular) lesions (table 2). Two were men. Two patients had a pontomedullary cavernoma, and another two had a discrete demyelinating lesion in the pons (figure 1). In a fifth patient (Patient 19), the pontine lesion was not imaged, but multiple periventricular demyelinating lesions typical of MS were identified on MR imaging. All five patients had neurologic symptoms and signs in addition to abduction paresis, but no other ocular motor signs. Two patients were tested acutely, that is, within 1 month of symptom onset. Serial eye movement recordings were performed on these two patients with acute, central (fascicular) palsy at presentation and at 2 months after symptom onset. The mean duration of diplopia (at the time of eye movement recording) in acute central palsies $(\mathrm{n}=2)$ was $0.38 \pm 0.18$ months, whereas the mean duration in chronic central palsies $(n=5)$ was $42 \pm$ 54 months. The duration of chronic peripheral palsies (mean $27 \pm 24$ months) was shorter than the duration of chronic central palsies (mean $42 \pm 54$ months), but the difference was not significant (Wilcoxon rank sum test). The mean age in acute central palsies $(\mathrm{n}=2)$ was $53 \pm 32$ years (median age $=53$ years; age range $=30$ to 75 years), whereas the mean age in chronic central palsies $(n=5)$ was $52 \pm 18$ years (median age $=56$ years; age range $=30$ to 75 years). The ages of the patients with central and peripheral palsies, acute or chronic, did not differ significantly ( $t$ tests).

All patients had a clinically obvious unilateral limitation of abduction (table 1), with an incomitant esotropia which increased in the field of action of the paretic muscle. 
Table 1 Characteristics of patients with peripheral sixth nerve palsy

\begin{tabular}{|c|c|c|c|c|c|c|c|c|}
\hline Patient & $\begin{array}{l}\text { Age, } \\
\text { y/sex }\end{array}$ & $\begin{array}{l}\text { Side of } \\
\text { lesion }\end{array}$ & Duration* & $\begin{array}{l}\text { Abduction deficit } \\
\text { as } \% \text { of normal* }\end{array}$ & Saccadic speed* & $\begin{array}{l}\text { Visual } \\
\text { acuity }\end{array}$ & Imaging & Comments \\
\hline 1 & $66 / \mathrm{M}$ & Right & $\begin{array}{l}3 \text { wk (acute) } \\
20 \text { mo (chronic) }\end{array}$ & $\begin{array}{l}80 \% \text { (acute) } \\
80 \% \text { (chronic) }\end{array}$ & $\begin{array}{l}\text { Slow (acute) } \\
\text { Normal (chronic) }\end{array}$ & $\begin{array}{l}\text { 20/40 OD; } \\
\text { 20/30 OS }\end{array}$ & Normal CT & $\mathrm{DM}$ \\
\hline 2 & $77 / \mathrm{M}$ & Right & $30 \mathrm{mo}$ & $60 \%$ & Normal & $\begin{array}{l}\text { 20/25 OD; } \\
\text { 20/30 OS }\end{array}$ & Normal MRI & Idiopathic \\
\hline 3 & $65 / \mathrm{M}$ & Left & $96 \mathrm{mo}$ & $70 \%$ & Normal & $20 / 20 \mathrm{OU}$ & Normal MRI & Idiopathic \\
\hline 4 & $50 / \mathrm{F}$ & Left & $24 \mathrm{mo}$ & $80 \%$ & Normal & $20 / 25 \mathrm{OU}$ & Normal MRI & Idiopathic \\
\hline 5 & $46 / \mathrm{M}$ & Left & $\begin{array}{l}3 \text { wk (acute) } \\
30 \text { mo (chronic) }\end{array}$ & $\begin{array}{l}80 \% \text { (acute) } \\
80 \% \text { (chronic) }\end{array}$ & $\begin{array}{l}\text { Slow (acute) } \\
\text { Normal (chronic) }\end{array}$ & $20 / 20 \mathrm{OU}$ & Normal MRI & HTN \\
\hline 6 & $64 / \mathrm{M}$ & Left & $\begin{array}{l}3 \mathrm{wk} \text { (acute) } \\
26 \mathrm{mo} \text { (chronic) }\end{array}$ & $\begin{array}{l}80 \% \text { (acute) } \\
80 \% \text { (chronic) }\end{array}$ & $\begin{array}{l}\text { Slow (acute) } \\
\text { Normal (chronic) }\end{array}$ & $\begin{array}{l}\text { 20/20 OD; } \\
20 / 25 \text { OS }\end{array}$ & Normal CT & HTN \\
\hline 7 & $75 / \mathrm{F}$ & Right & $4 \mathrm{mo}$ & $90 \%$ & Normal & $20 / 40 \mathrm{OU}$ & Normal MRI & Idiopathic \\
\hline 8 & $77 / \mathrm{F}$ & Right & $10 \mathrm{mo}$ & $95 \%$ & Normal & $\begin{array}{l}\text { 20/30 OD; } \\
\text { 20/20 OS }\end{array}$ & Normal MRI & Idiopathic \\
\hline 9 & $52 / \mathrm{M}$ & Right & $\begin{array}{l}3 \text { wk (acute) } \\
17 \text { mo (chronic) }\end{array}$ & $\begin{array}{l}95 \% \text { (acute) } \\
95 \% \text { (chronic) }\end{array}$ & $\begin{array}{l}\text { Slow (acute) } \\
\text { Normal (chronic) }\end{array}$ & $20 / 20 \mathrm{OU}$ & Normal MRI & Idiopathic \\
\hline 10 & $64 / \mathrm{M}$ & Right & $15 \mathrm{mo}$ & $90 \%$ & Normal & $\begin{array}{l}\text { 20/20 OD; } \\
20 / 25 \text { OS }\end{array}$ & Normal CT & Idiopathic \\
\hline 11 & $57 / \mathrm{M}$ & Right & $\begin{array}{l}3 \text { wk (acute) } \\
23 \text { mo (chronic) }\end{array}$ & $\begin{array}{l}90 \% \text { (acute) } \\
90 \% \text { (chronic) }\end{array}$ & $\begin{array}{l}\text { Slow (acute) } \\
\text { Normal (chronic) }\end{array}$ & $20 / 25 \mathrm{OU}$ & Normal CT & HTN, DM \\
\hline 12 & $57 / \mathrm{M}$ & Left & $34 \mathrm{mo}$ & $90 \%$ & Normal & $\begin{array}{l}\text { 20/30 OD; } \\
\text { 20/25 OS }\end{array}$ & Normal MRI & Idiopathic \\
\hline 13 & $75 / \mathrm{F}$ & Left & $12 \mathrm{mo}$ & $90 \%$ & Normal & $20 / 25 \mathrm{OU}$ & Normal MRI & Idiopathic \\
\hline 14 & $54 / \mathrm{F}$ & Left & $60 \mathrm{mo}$ & $80 \%$ & Normal & $\begin{array}{l}\text { 20/25 OD; } \\
\text { 20/20 OS }\end{array}$ & Normal MRI & Idiopathic \\
\hline
\end{tabular}

\footnotetext{
* Duration of palsy, abduction deficit, and saccadic speed at the time of eye movement recording (patients with serial recordings have two values: one during acute and one during chronic state, as specified. All other patients have one recording only in the chronic state).
}

$\mathrm{OD}=$ right eye; $\mathrm{OS}=$ left eye; $\mathrm{DM}=$ diabetes mellitus; $\mathrm{OU}=$ bilaterally; HTN $=$ hypertension.

In peripheral palsies, the mean abduction deficit (as percentage of normal abduction) in the acute state $(\mathrm{n}=5)$ was $85 \pm 7 \%$, whereas the mean abduction deficit in the chronic state $(\mathrm{n}=14)$ was $84 \pm 10 \%$. In central palsies, the mean abduction deficit in the acute state $(\mathrm{n}=2)$ was $70 \%$, whereas the mean abduction deficit in the chronic state $(\mathrm{n}=5)$ was $79 \pm 7 \%$. The severity of the abduction deficit in chronic peripheral (mean 84\%) vs chronic central palsies (mean 79\%) did not differ ( $p=0.36, t$ test).

Saccades in peripheral abducens nerve palsy. Figure 2A shows representative recordings of horizontal saccades made by Patient 1 who had a peripheral palsy, while viewing with the paretic right eye during the acute state (3 weeks after onset of symptom). During saccades from center to the paretic orbital hemi-range of duction (i.e., from center to $10 \mathrm{deg}$ right), centrifugal abducting peak velocity of the paretic right eye was reduced, when compared with normal controls (Z-test, $p<0.05$ ). Each of the five patients with recordings in the acute state had slow centrifugal abducting saccades with prolonged duration (Z-tests, $p<$ 0.05). The mean (group) peak velocity-amplitude and duration-amplitude relationships of initial saccades in acute peripheral palsy are shown in figures $3 \mathrm{~A}$ and $4 \mathrm{~A}$. We correlated peak velocities and durations with bins of amplitude in 2 deg ranges because the palsies precluded measuring large amplitude saccades to determine asymptotic features of the peak velocity-amplitude main sequence in our patients. As a group, acute peripheral palsy $(\mathrm{n}=5)$ had reduced peak velocities and longer durations for centrifugal abducting saccades for any given amplitude $(p<0.05)$.

Figure 2B shows representative recordings of horizontal saccades made by the same patient (Patient 1 ) during the chronic state. Peak velocity of centrifugal abducting saccades of the paretic right eye returned to normal when recorded 2 months after onset of symptoms. In each of five acute palsies tested serially, saccade peak velocities and durations became normal within 2 months of symptom onset despite persisting limited abduction (i.e., severity of the abduction deficit in the acute and chronic states was the same in all five patients). Saccade peak velocities and durations were also normal in each of nine other patients who had recording in the chronic state only. As shown in figures $3 \mathrm{~A}$ and $4 \mathrm{~A}$, as a group, chronic peripheral palsy ( $\mathrm{n}=14$ ) had normal peak velocities and durations for centrifugal abducting saccades, when compared with normal controls $(p>0.1)$.

Centripetal abducting saccades from 10 deg adducted position to center in the paretic eye had normal peak velocity and duration, both in acute and chronic states in all patients. Saccade peak velocities and durations in the nonparetic eye were normal in both acute and chronic states in all patients. 
Table 2 Characteristics of patients with central fascicular sixth nerve palsy

\begin{tabular}{|c|c|c|c|c|c|c|c|c|}
\hline Patient & $\begin{array}{l}\text { Age, } \\
\text { y/sex }\end{array}$ & $\begin{array}{l}\text { Side of } \\
\text { lesion }\end{array}$ & Duration* & $\begin{array}{l}\text { Abduction } \\
\text { deficit as \% } \\
\text { of normal* }\end{array}$ & $\begin{array}{l}\text { Saccadic } \\
\text { speed* }^{*}\end{array}$ & $\begin{array}{l}\text { Visual } \\
\text { acuity }\end{array}$ & Imaging & Presenting symptoms \\
\hline 17 & $56 / \mathrm{F}$ & Left & $132 \mathrm{mo}$ & $70 \%$ & Slow & $\begin{array}{l}20 / 25 \text { OD } \\
20 / 30 \text { OS }\end{array}$ & $\begin{array}{l}\text { MRI: Left pontomedullary } \\
\text { cavernoma }\end{array}$ & $\begin{array}{l}\text { Left facial palsy and } \\
\text { paresthesia }\end{array}$ \\
\hline 18 & $30 / \mathrm{F}$ & Left & $\begin{array}{c}2 \mathrm{wk} \\
\text { (acute) } \\
15 \mathrm{mo} \\
\text { (chronic) }\end{array}$ & $\begin{array}{l}70 \% \text { (acute) } \\
80 \% \text { (chronic) }\end{array}$ & $\begin{array}{l}\text { Slow (acute) } \\
\text { Slow (chronic) }\end{array}$ & $20 / 25 \mathrm{OU}$ & $\begin{array}{l}\text { MRI: Left pontomedullary } \\
\text { demyelinating lesion }\end{array}$ & Diplopia, ataxia \\
\hline
\end{tabular}

\footnotetext{
* Duration of palsy, abduction deficit, and saccadic speed at the time of eye movement recording (patients with serial recordings have two values: one during acute and one during chronic state, as specified. All other patients have one recording only in the chronic state).
}

$\mathrm{OU}=$ bilaterally; $\mathrm{MS}=$ multiple sclerosis; $\mathrm{OD}=$ right eye; $\mathrm{OS}=$ left eye.

Saccades in central fascicular abducens nerve palsy. Figure 5A shows representative recordings of horizontal saccades made by Patient 15 who had a central fascicular
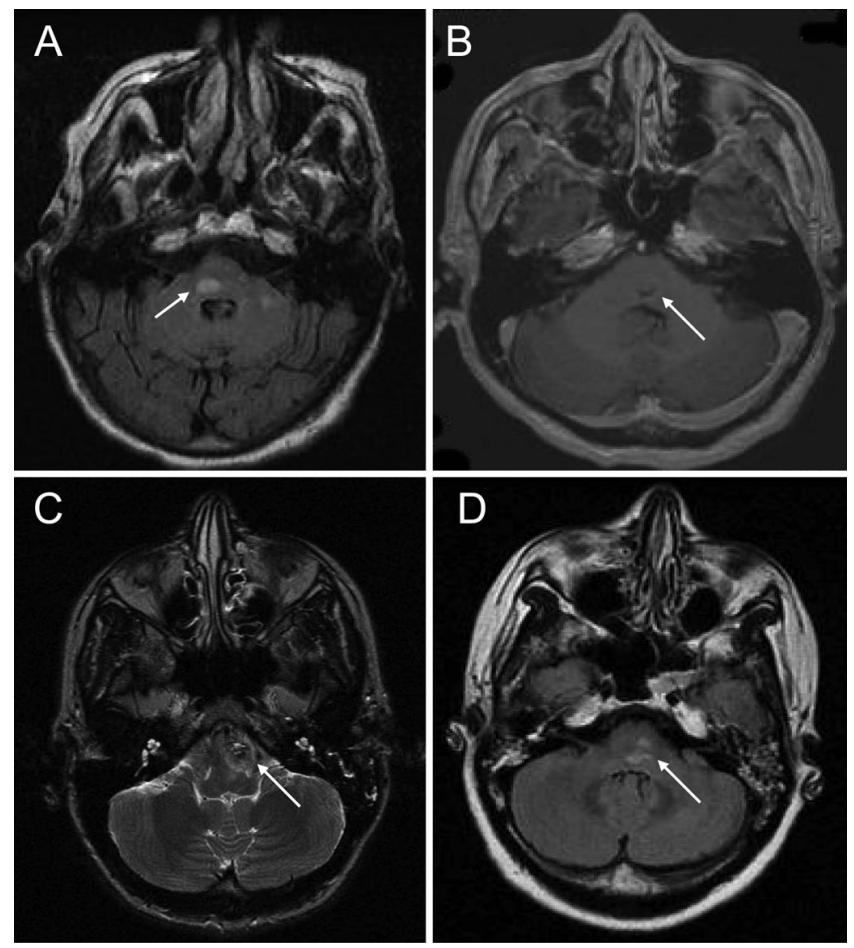

Figure 1. MRI of patients with central (fascicular) sixth nerve palsy. (A) Patient 15. FLAIR image showing a demyelinating lesion in the right pons. (B) Patient 16. T2weighted image with gadolinium enhancement showing a left pontomedullary cavernoma and hematoma. (C) $\mathrm{Pa}$ tient 17. T2-weighted image showing a left pontomedullary cavernoma. (D) Patient 18. FLAIR image showing a demyelinating lesion in the left pontomedullary area. palsy, while viewing with the paretic right eye during the acute state ( 1 week after onset of symptom). Peak velocity of centrifugal abducting saccades (i.e., from center to 10 deg right) of the paretic right eye was reduced, when compared with normal controls. In each of two patients with recording in the acute state, centrifugal abducting saccades in the paretic eye had reduced peak velocities (figure $3 \mathrm{~B}$ and longer durations (figure $4 \mathrm{~B}$ for any given amplitude (Z-tests, $p<0.05$ ).

Figure 5B shows representative recordings of horizontal saccades made by the same patient (Patient 15) during the chronic state. Peak velocity of centrifugal abducting saccades of the paretic right eye remained reduced when recorded at 2 months after onset of symptom. Similar observation was made in the second patient with serial recordings, despite the fact that the severity of abduction deficit in these two patients improved in the chronic state. Saccade peak velocities and durations were also abnormal in each of three other patients who had recording in the chronic state only. As shown in figures $3 \mathrm{~B}$ and $4 \mathrm{~B}$, as a group, chronic central palsy $(\mathrm{n}=5)$ had reduced peak velocities and prolonged durations for centrifugal abducting saccades, when compared with normal controls $(p>0.1)$.

Centripetal abducting saccades from $10 \mathrm{deg}$ adducted position to center in the paretic eye had normal peak velocity and duration, both in acute and chronic states in all patients. Saccade peak velocities and durations in the nonparetic eye were normal in both acute and chronic states in all patients.

Discussion. Information about saccadic abnormalities in paralytic strabismus is sparse. A report ${ }^{11}$ of three patients with fascicular sixth nerve palsies localized by MRI identified low saccadic velocities in the paretic eye on abduction. In our laboratory, seven patients with unilateral peripheral sixth or third nerve palsies were found by magnetic search 
A Acute
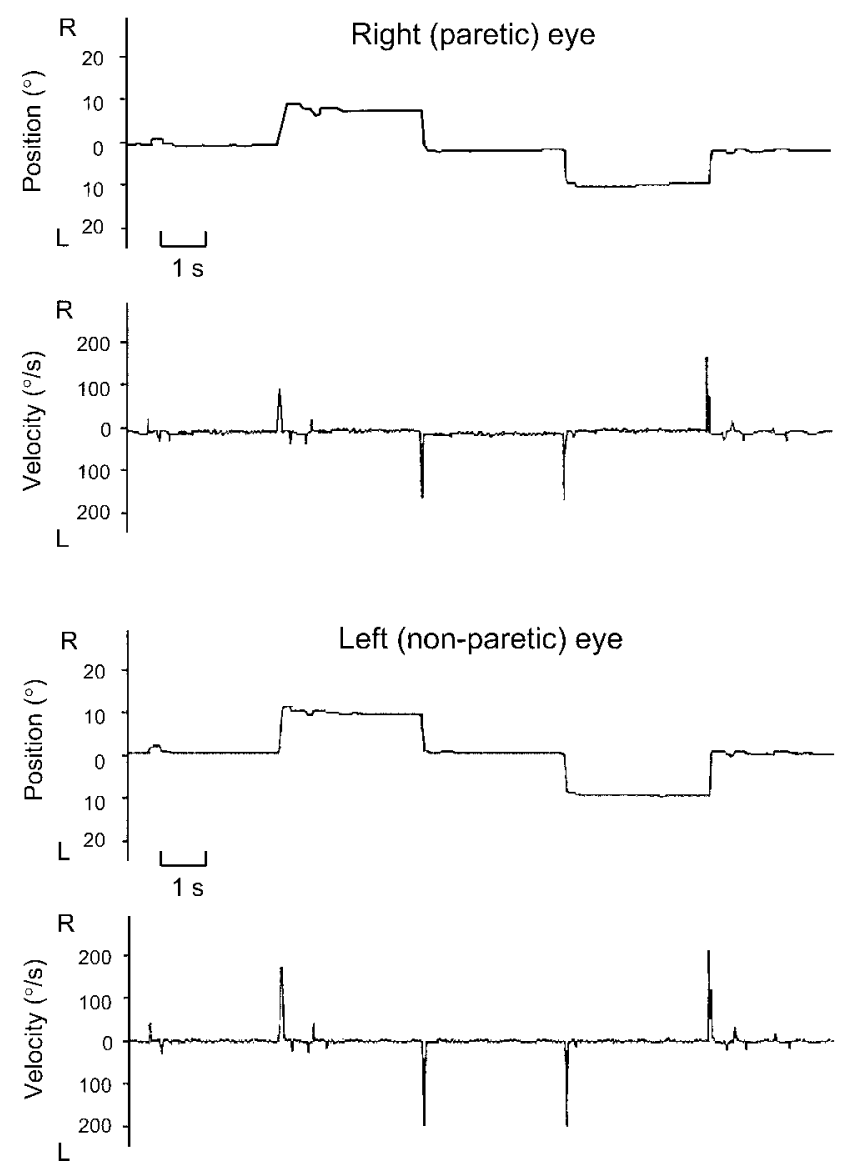

B Chronic
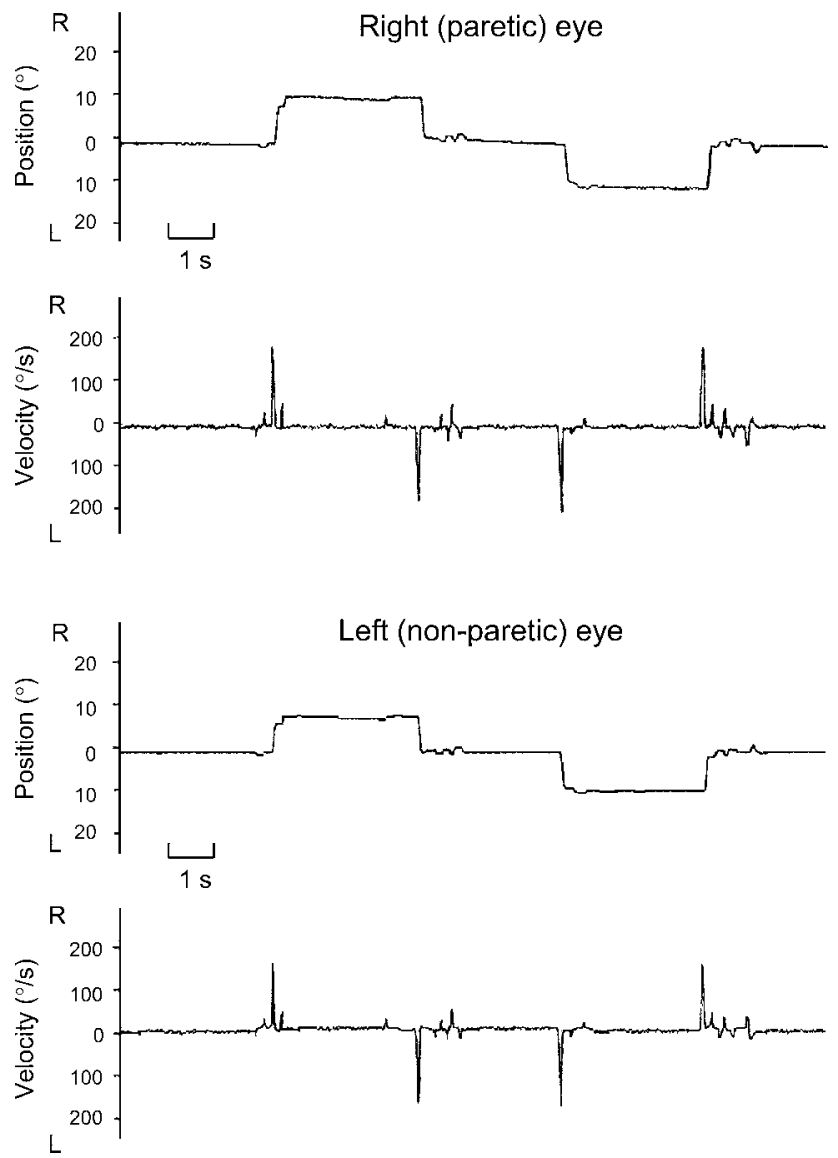

Figure 2. Serial recordings of horizontal saccades made by Patient 1 who had a right peripheral sixth nerve palsy, while viewing with the paretic right eye. (A) Recordings obtained 3 weeks after symptom onset (acute palsy). (B) Repeated recordings at 2 months (chronic palsy). During saccades from center to the paretic orbital hemi-range of duction (i.e., from center to 10 deg right), centrifugal abducting saccadic peak velocity of the paretic right eye was low during acute state, but it became normal during chronic state.

coil technique to have reduced peak velocities in the direction of paretic agonists. ${ }^{12}$ Slowing of saccades was also reported in single cases or a few patients with unilateral peripheral sixth nerve palsy or medial rectus palsy. ${ }^{1-4,13-15}$ The effects of duration (acute vs chronic) and site (peripheral vs central) of abducens nerve palsy on saccade dynamics had not been systematically investigated, nor had the potential of recovery been previously addressed.

In this investigation, we measured the effects of idiopathic, presumed ischemic, peripheral palsy, and central fascicular palsy on the peak velocity and duration of saccades, and assessed changes over time. Although abducting saccades are hypometric and slow in the paretic eye in lateral rectus palsy, ${ }^{1-4,12,15}$ we measured saccade speeds well within the range of limited abduction, and saccades were not hypometric within this range (table E-1 on the Neurology Web site at www.neurology.org). Acutely, centrifugal abducting saccades in the paretic orbital hemi-range of duction (from center to an abducted position) in the paretic eye are slow in both central and peripheral palsy, as anticipated from weakness of the lateral rectus muscle. In chronic state, centrifugal abducting saccadic peak velocities remain reduced and durations remain prolonged in the paretic eye in central palsy, but they become normal in peripheral palsy. If chronic central palsies had greater severity than chronic peripheral palsy, the results might be biased toward more recovery in the peripheral palsy. However, there was no significant difference in the mean severity of abduction limitation between central and peripheral palsies. Centripetal abducting saccades from an adducted position to center in the paretic eye, as well as adducting saccades in the non-paretic eye (field of action of the contralateral yoked medial rectus muscle), were normal in amplitude, velocity, and duration in both central and peripheral palsy, acute or chronic.

In sixth nerve palsy, abduction is little affected when the eye starts from an adducted (nasal) position, since most of the change in force is contributed by relaxation of the normal antagonist, that is, the medial rectus. ${ }^{16}$ When the movement begins from orbital mid-position, however, the change in force comes mainly from contraction of the agonist, ${ }^{16}$ that 


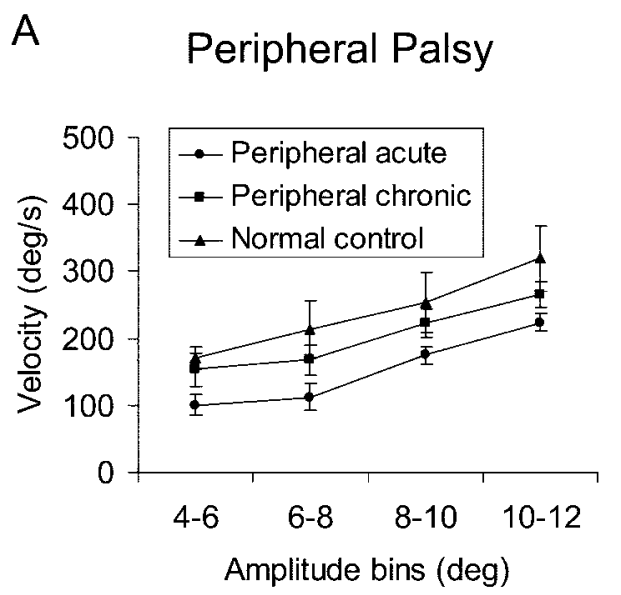

\section{B Central (Fascicular) Palsy}

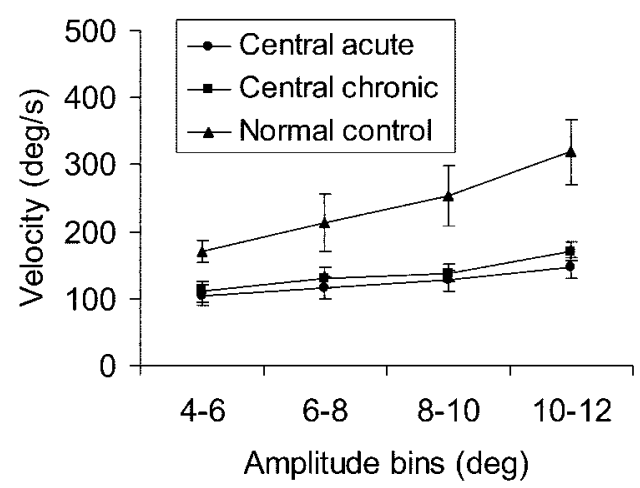

Figure 3. Mean (group) peak velocity vs amplitude (in bins) of initial centrifugal abducting saccades from center to 10 deg abducted position in the paretic eye. (A) Peripheral palsy. (B) Central (fascicular) palsy. Error bars indicate $1 \mathrm{SD}$ from the normal group mean.

is, the paretic lateral rectus, and hence saccades in the paretic orbital hemi-range of duction are abnormal. Our finding that centrifugal abducting saccades in the paretic orbital hemi-range of duction (from center to an abducted position) in the paretic eye are slow, while centripetal abducting saccades from an adducted position to center in the paretic eye are normal, can be explained by the relative contribution of the agonist and antagonist muscles in different orbital positions.

In paralytic strabismus, "contracture" (shortening and increased stiffness) occurs in the antagonist of the paretic muscle, ${ }^{17-20}$ while the paretic muscle lengthens in response to a change in orbital position of the globe. Contracture of the non-paretic antagonist is associated with a decrease in the number of sarcomeres, whereas lengthening of the paretic muscle is accompanied by an increase in sarcomeres. ${ }^{21}$ The persistent reduction in centrifugal abducting saccadic velocities found in our patients with chronic central palsy is unlikely to be the result of contracture of the medial rectus (antagonist) muscle because such contracture might be expected to affect
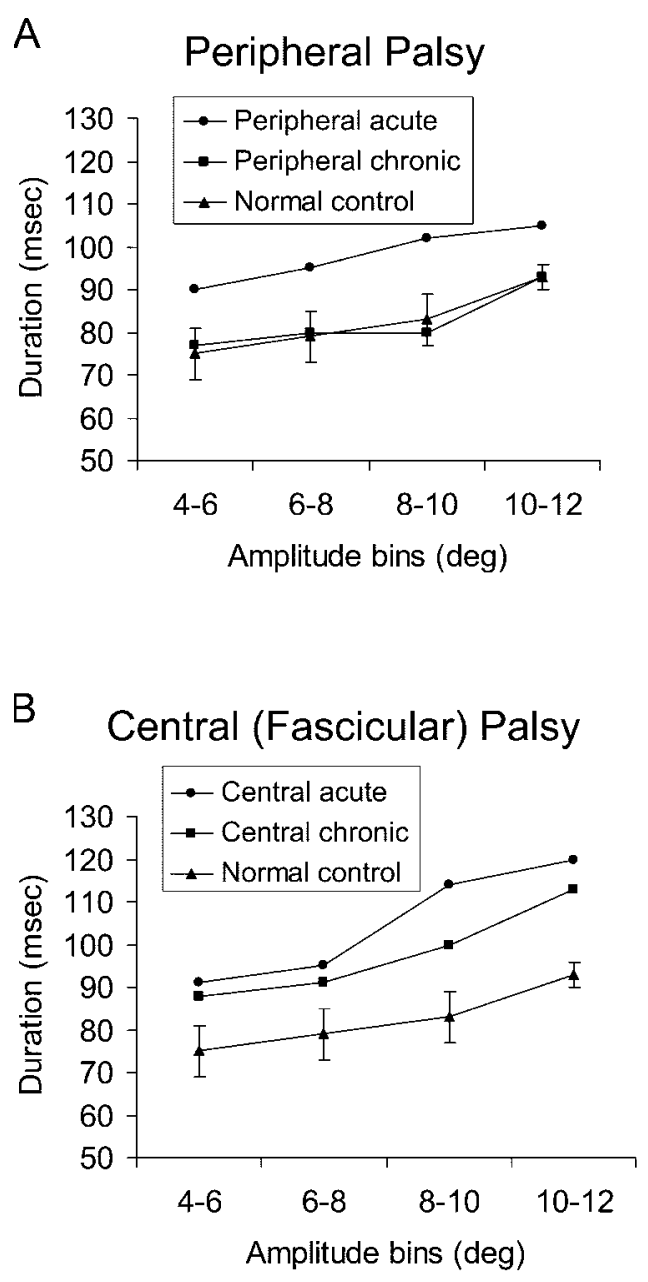

Figure 4. Mean (group) duration vs amplitude (in bins) of initial centrifugal abducting saccades from center to 10 deg abducted position in the paretic eye. (A) Peripheral palsy. (B) Central (fascicular) palsy. Error bars indicate 1 $S D$ from the normal group mean.

centripetal abducting saccades (from adducted hemirange to orbital mid-position), by reducing its relaxation or length; however, they were normal in our patients.

The abducens is the motor nerve with the most substantial course both within and outside the brain, where it is vulnerable to damage. ${ }^{22}$ Comparison of recovery after central vs peripheral nerve palsy provides a unique opportunity to investigate differences in repair properties ${ }^{23}$ between the central and peripheral nervous systems. We found that in chronic peripheral palsy, abducting saccadic peak velocity and duration for the tested range of excursion (10 deg) become normal within 2 months after symptom onset, despite persistent abduction deficit and esotropia. In contrast, there was no improvement in saccadic peak velocity or duration in chronic central fascicular palsy. Our findings are concordant with the capacity for recovery of peripheral nerves and also with the limited capacity for recovery of central nerves. ${ }^{23}$ The relatively rapid time course for recovery of saccadic speed after peripheral palsy in our 
A Acute
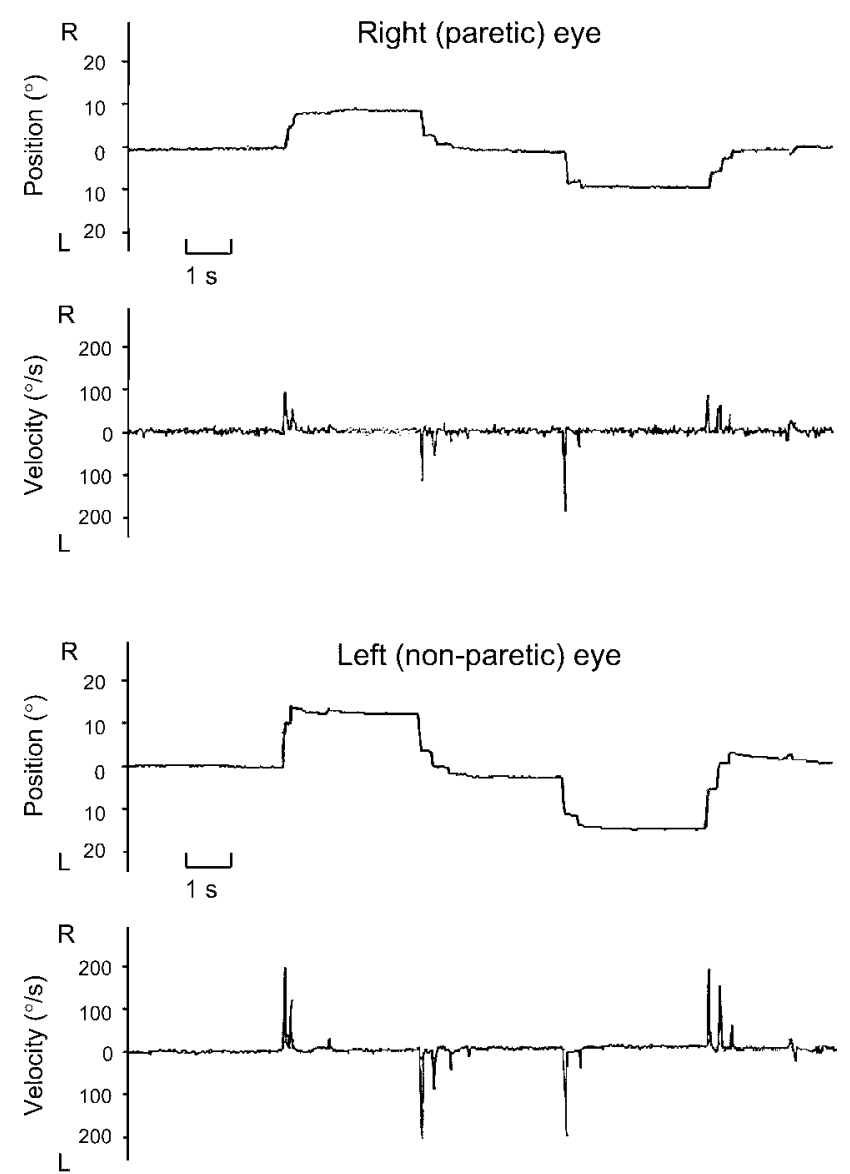

B Chronic
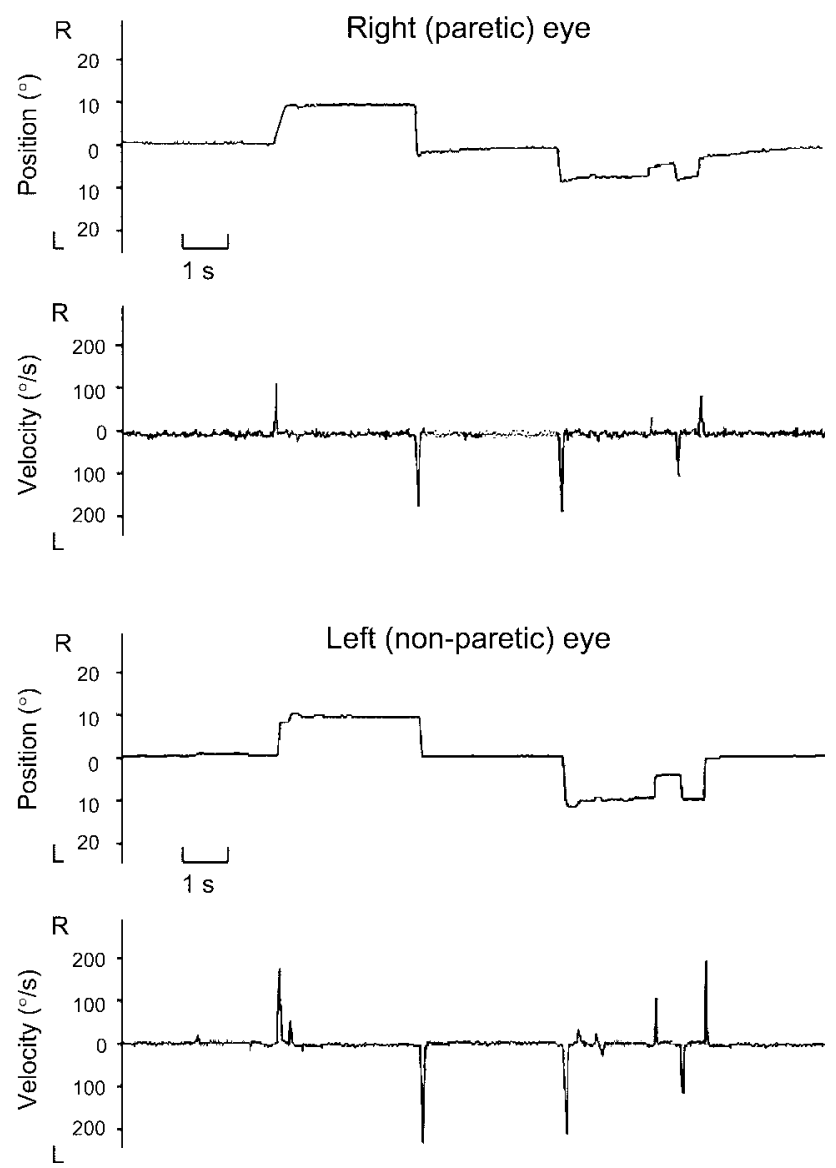

Figure 5. Serial recordings of horizontal saccades made by Patient 15 who had a right central (fascicular) palsy, while viewing with the paretic right eye. (A) Recordings obtained 3 weeks after symptom onset (acute palsy). (B) Repeated recordings at 2 months (chronic palsy). Unlike patients with peripheral palsy, centrifugal abducting velocities for saccades from center to the paretic orbital hemi-range of duction (i.e., from center to 10 deg right) of the paretic right eye were low during both acute and chronic states.

patients is consistent with demyelinating damage, or neurapraxia, ${ }^{24-26}$ followed by remyelination.

Histopathologic correlation for ocular motor nerve palsies is sparse. ${ }^{27-30}$ The oculomotor nerve of one diabetic patient with a third nerve palsy showed mild disruption of the myelin sheaths and axis cylinders in the center of the nerve, providing evidence for a functional block rather than axonal disruption. ${ }^{27}$ Two subsequent studies ${ }^{28,29}$ of oculomotor nerve palsy in two diabetic patients also found the lesion to be primarily demyelinating, with a paucity of Wallerian degeneration of axons distally, and an absence of chromatolysis in neuronal cell bodies in the oculomotor nucleus. These findings ${ }^{27-29}$ were compatible with the rapid and complete recovery of ocular motor nerve palsy commonly seen in diabetes.

No histopathologic study of diabetic, hypertensive, or idiopathic (presumed microvascular ischemic) sixth nerve palsy is available. However, a feline model using axotomy of the abducens nerve shows that the time course for structural and functional recovery is about 3 months. ${ }^{30}$ In our study, we found that abducting saccadic peak velocity and duration became normal within 2 months in patients with idiopathic, presumably ischemic, peripheral palsy. Although the range of abduction remained limited in the peripheral sixth nerve palsies we studied, in contrast to the typical complete recovery of abduction after idiopathic (presumed microvascular ischemic) palsies, the time course of recovery of saccade speed to normal in these patients suggests that their palsies resulted from a functional or primarily demyelinating event (first-degree injury), rather than from axonal disruption (second-degree injury). ${ }^{24-26}$

A high frequency burst of phasic discharge in agonist motoneurons, the saccadic pulse, drives the eyes at high speed, ${ }^{31,32}$ and a level of tonic innervation, constituting saccadic step of innervation, holds the eye in its new position. ${ }^{31-33}$ In peripheral palsy, abducting saccadic peak velocity and duration became normal, in face of persistent esotropia. We postulate that the partially recovered peripheral nerve behaves like a high pass filter, allowing the transmission of high firing rates during the pulse of innervation, such that the resulting saccades are of normal peak velocity and duration. In contrast, dur- 
ing the step of innervation, the partially recovered peripheral nerve may be unable to transmit low frequency tonic signals, resulting in abnormal tonic position (i.e., esotropia) of the paretic eye. This is the converse of the Wedensky phenomenon, ${ }^{34,35}$ whereby high frequency nerve discharges through partially damaged nerve fibers are attenuated while low frequency discharges are preserved.

A cellular correlate of disparity between the repair of saccade speed and the persistence of defective ranges of abduction may be the selective nature of the nerve fibers damaged, or the tempo of their regeneration or remyelination. Although all ocular motoneurons participate in saccadic and slow eye movements, and in the tonic discharges that maintain eye positions, ${ }^{36,37}$ some may be specialized in function. Large motoneurons within the abducens nucleus innervate singly innervated, twitch muscle fibers, whereas smaller motoneurons around its periphery innervate multiply innervated, non-twitch muscle fibers. ${ }^{38,39}$ The high pass filter response of the damaged peripheral abducens nerve to saccadic peak velocity commands, as described above, might be explained by either predominant damage to peripheral nerve fibers from "non-twitch" motoneurons just outside the abducens nucleus, or by better repair of nerve fibers from "twitch" motoneurons within the nucleus.

We found that abducting saccadic peak velocity remained reduced in patients with central palsy from cavernomas or MS. Although MS is generally considered to be an inflammatory demyelinating disease with relative axonal sparing, axonal damage, including loss, transection, or disturbed axonal transport, occurs early in the disease and during lesion development. ${ }^{40-44}$ The absence of recovery of saccade speed and range in our patients with central fascicular palsy was consistent with limited regeneration of damaged axons or myelin in the glial environment of the CNS. ${ }^{23,45-51}$

In chronic peripheral palsy, peak velocities of abducting saccades in the paretic eye became normal. Their normal speed may be due to partial recovery of the peripheral nerve, as discussed above. Recovery may also represent a monocular readjustment of innervation selectively to the paretic eye. If common and conjugate premotor signals were sent to motoneurons to both eyes, adducting saccadic velocity in the yoked antagonist of the non-paretic eye would be increased as well, in accord with Hering's propos$\mathrm{al}^{52}$ that yoked muscles of the two eyes are always equally innervated by common conjugate commands so that one eye is not moved independently of the other. For example, in the case of a left lateral rectus weakness from a left sixth nerve palsy, any adaptive increase in innervation to the left lateral rectus would be accompanied by increased innervation to the right medial rectus, in accord with Hering's proposal or "law." However, this was not the case; yoked adduction of the fellow eye had normal peak velocityamplitude relationship. Monocular adaptation of sac- cades to sixth nerve palsy would be consistent with evidence of monocular adaptation of the vestibuloocular reflex after sixth, fourth, or third nerve palsy. ${ }^{53-55}$

In monkeys, tenotomy of both horizontal rectus muscles of one eye reduces saccade amplitude in that eye, and when the normal eye is occluded, there is a conjugate increase in saccade amplitude in both eyes, obeying Hering's law. ${ }^{5}$ After the patch is removed, saccade amplitudes are reduced in the normal eye to match those of the paretic eye, signifying monocular adaptation to the normal eye, ${ }^{5}$ and perhaps the paretic eye. Small or moderate weakening of one horizontal rectus muscle in monkeys, followed by patching of that eye, ${ }^{6}$ results in selective monocular increase in eye movement amplitude in the operated eye only, suggesting monocular adjustments. ${ }^{6}$ We did not assess the effects of monocular occlusion in this study. Our patients had mild-to-moderate palsy; they viewed with both eyes but habitually fixated with their non-paretic eye. We observed a monocular unidirectional change in saccade speed, within the range of spared amplitudes (duction) in the paretic eye after peripheral palsy, but not after central palsy.

An anatomic and physiologic pre-nuclear substrate for possible monocular adjustments of saccade speed in response to peripheral nerve palsy is found in pre-saccadic burst neurons of the caudal paramedian pontine reticular formation (PPRF). The great majority (about $80 \%$ ) of short-lead burst neurons in the caudal PPRF of monkeys that were once thought to encode conjugate velocity commands for horizontal saccades actually encode monocular movements of either abduction or adduction. ${ }^{56}$ This behavior is contrary to Hering's "law." Whether the present results indicate partial recovery, monocular adaptation, or both, the normalization of saccadic speed in chronic peripheral palsy allows both eyes to reach targets in the paretic hemifield of motion rapidly and simultaneously. Persisting slowing of saccades after central palsies may reflect damage to mechanisms of monocular adaptation, in addition to limited regeneration of axons or myelin within the brain.

Idiopathic abducens nerve palsy in patients over 50 years of age is usually presumed to be caused by ischemia from arteriolosclerosis of small vessels, occurring with greater frequency in patients with diabetes mellitus or hypertension. ${ }^{57}$ Since most patients recover within 3 months, ${ }^{58}$ they may not require imaging of the brain or skull base at the time of initial presentation, ${ }^{10}$ if they have no other neurologic symptoms or signs. However, if there is no improvement within 3 months, imaging studies are indicated. Assessment of abducting saccadic velocity may be an adjunct to imaging in distinguishing between a central and peripheral cause of abducens nerve palsy. Study of palsies having comparable severity after partial structural damage to the peripheral nerve from compression or infiltration will be needed to determine if their saccadic dynamics differ from 
those in idiopathic (presumed ischemic) peripheral palsies or from central palsies, as investigated here. Normal saccadic velocity after 2 months may specify peripheral nerve damage presumed to be ischemic in origin. Conversely, reduced saccadic velocity after 2 months might signify a central lesion. In this situation, prompt imaging studies are warranted to detect a brainstem lesion or extra-axial mass lesion as the cause of paretic abduction.

\section{References}

1. Metz HS, Scott AB, O'Meara D, Stewart HL. Ocular saccades in lateral rectus palsy. Arch Ophthalmol 1970;84:453-460.

2. Kommerell G, Olivier D, Theopold H. Adaptive programming of phasic and tonic components in saccadic eye movements: investigations in patients with abducens palsy. Invest Ophthalmol 1976;15:657-660.

3. Zee DS, Yee RD. Abnormal saccades in paralytic strabismus. Am J Ophthalmol 1977;83:112-114.

4. Optican LM, Zee D, Chu F. Adaptive response to ocular muscle weakness in human pursuit and saccadic eye movements. J Neurophysiol 1985;54:110-122.

5. Snow R, Hore J, Vilis T. Adaptation of saccadic and vestibulo-ocular systems after extraocular muscle tenectomy. Invest Ophthalmol Vis Sci 1985;26:924-931.

6. Viirre E, Cadera W, Vilis T. Monocular adaptation of the saccadic system and vestibulo-ocular reflex. Invest Ophthalmol Vis Sci 1988;29: 1339-1347.

7. Sharpe JA, McReelis K, Wong AM. Recovery of peripheral versus central nerves identified by saccadic velocity after abducens neuropathy. Ann NY Acad Sci 2005;1039:417-429.

8. Savitzky A, Golay MJE. Smoothing and differentiation of data by simplified least squared procedures. Analytical Chemistry 1964;8:16271639.

9. Sargent JC. Nuclear and infranuclear ocular motility disorders. In: Miller NR, Newman NJ, Biousse V, Kerrison JB, eds. Walsh and Hoyt's clinical neuro-ophthalmology. 6th ed. Philadelphia: Lippincott Williams \& Wilkins, 2005; 969-1040.

10. Chou KL, Galetta SL, Liu GT, et al. Acute ocular motor mononeuropathies: prospective study of the roles of neuroimaging and clinical assessment. J Neurol Sci 2004;219:35-39.

11. Bronstein AM, Morris J, Du Boulay G, Gresty MA, Rudge P. Abnormalities of horizontal gaze. Clinical, oculographic and magnetic resonance imaging findings. I Abducens Palsy J Neurol Neurosurg Psychiatry 1990;53:194-199.

12. Barton JJ, Huaman AG, Sharpe JA. Effects of edrophonium on saccadic velocity in normal subjects and myasthenic and nonmyasthenic ocular palsies. Ann Neurol 1994;36:585-594.

13. Theopold HGK. Phasic and tonic function of eye muscles. Saccades in patients with oculomotor or abducens paralysis. Albrecht Von Graefes Arch Klin Exp Ophthalmol 1974;192:247-254.

14. Abel LA, Schmidt D, Dell'Osso LF, Daroff RB. Saccadic system plasticity in humans. Ann Neurol 1978;4:313-318.

15. Leigh RJ, Zee DS. The neurology of eye movements. 3rd ed. New York: Oxford University Press, 1999.

16. Collins CC. The human oculomotor control system. In: Lennerstrand G, Bach-y-Rita P, eds. Basic mechanisms of ocular motility and their clinical implications. New York: Pergamon Press, 1975;145-180.

17. Jampolsky A. A simplified approach to strabismus diagnosis. In: Symposium on Strabismus; Transactions of the New Orleans Academy of Ophthalmology. St. Louis: C.V. Mosby, 1971;34-92.

18. Scott AB. Active force tests in lateral rectus paralysis. Arch Ophthalmo 1971;85:397-404

19. Scott AB, Collins CC, O'Meara DM. A forceps to measure strabismus forces. Arch Ophthalmol 1972;88:330-333.

20. Scott AB, Kraft SP. Botulinum toxin injection in the management of lateral rectus paresis. Ophthalmology 1985;92:676-683.

21. Scott AB. Change of eye muscle sarcomeres according to eye position. J Pediatr Ophthalmol Strabismus 1994;31:85-88.

22. Sharpe JA, Wong AM. Anatomy and physiology of ocular motor systems. In: Miller NR, Newman NJ, Biousse V, Kerrison JB, eds. Walsh and Hoyt's clinical neuro-ophthalmology. 6th ed. Philadelphia: Lippincott Williams \& Wilkins, 2005;809-885.

23. Fenrich K, Gordon T. Axonal regeneration in the peripheral and central nervous systems-current issues and advances. Can J Neurol Sci 2004; 31:142-156

24. Seddon HJ. Three types of nerve injury. Brain 1943;66:237.

25. Sunderland S. Some anatomical and pathophysiological data relevant to the facial nerve injury and repair. In: Fisch U, ed. Facial nerve surgery. Amstelveen: Kugler, 1977;47-61.
26. Schaumberg HH, Berger AR, Thomas PK. Disorders of peripheral nerves. Philadelphia: FL Davis, 1992.

27. Dreyfus PM, Hakim S, Adams RD. Diabetic ophthalmoplegia; report of case, with postmortem study and comments on vascular supply of human oculomotor nerve. AMA Arch Neurol Psychiatry 1957;77:337-349.

28. Weber RB, Daroff RB, Mackey EA. Pathology of oculomotor nerve palsy in diabetics. Neurology 1970;20:835-838.

29. Asbury AK, Aldredge H, Hershberg R, Fisher CM. Oculomotor palsy in diabetes mellitus: a clinico-pathological study. Brain 1970;93:555-566.

30. Delgado-Garcia JM, Del Pozo F, Spencer RF, Baker R. Behavior of neurons in the abducens nucleus of the alert cat-III. Axotomized motoneurons. Neuroscience 1988;24:143-160.

31. Robinson DA. The mechanics of human saccadic eye movement. J Physiol (Lond) 1964;174:245-264.

32. Robinson DA. Oculomotor unit behavior in the monkey. J Neurophysiol 1970;33:393-404.

33. Bahill AT, Hsu FK, Stark L. Glissadic overshoots are due to pulse width errors. Arch Neurol 1978;35:138-142.

34. Lorente de No R. A study of nerve physiology. Studies from the Rockefeller Institute for Medical Research 1947;131:39-40.

35. Tasaki I. Nervous transmission. Springfield, IL: Charles C. Thomas Publisher, 1953.

36. Scott AB, Collins CC. Division of labor in human extraocular muscles. Arch Ophthalmol 1973;90:319-322.

37. Robinson DA. The functional behavior of the peripheral ocular motor apparatus: a review. In: Kommerell G, ed. Disorders of ocular motility. Munich: Bergman Verlag, 1978;43-61.

38. Buttner-Ennever JA, Horn AKE, Scherberger H, D'Ascanio P. Motoneurons of twitch and nontwitch extraocular muscle fibers in the abducens, trochlear, and oculomotor nuclei of monkeys. J Comp Neurol 2001;438: 318-335.

39. Buttner-Ennever JA, Eberhorn A, Horn AK. Motor and sensory innervation of extraocular eye muscles. Ann NY Acad Sci 2003;1004:40-49.

40. Ferguson B, Matyszak MK, Esiri MM, Perry VH. Axonal damage in acute multiple sclerosis lesions. Brain 1997;120:393-399.

41. Mews I, Bergmann M, Bunkowski S, Gullotta F, Brück W. Oligodendrocyte and axon pathology in clinically silent multiple sclerosis lesions. Mult Scler 1998;4:55-62.

42. Trapp BD, Peterson J, Ransohoff RM, Rudick R, Mork S, Bo L. Axonal transection in the lesions of multiple sclerosis. N Engl J Med 1998;338: $278-285$.

43. Lovas G, Szilágyi N, Komoly S, Palkovits M, Komoly S. Axonal changes in chronic demyelinated cervical spinal cord plaques. Brain 2000;123: 308-317.

44. Kornek B, Storch MK, Bauer J, et al. Distribution of a calcium channel subunit in dystrophic axons in multiple sclerosis and experimental autoimmune encephalomyelitis. Brain 2001;124:1114-1124.

45. George R, Griffin JW. Delayed macrophage responses and myelin clearance during Wallerian degeneration in the central nervous system: the dorsal radiculotomy model. Exp Neurol 1994;129:225-236.

46. Qiu J, Cai D, Filbin MT. Glial inhibition of nerve regeneration in the mature mammalian CNS. Glia 2000;29:166-174.

47. Bandtlow CE, Schwab ME. NI-35/250/nogo-a: a neurite growth inhibitor restricting structural plasticity and regeneration of nerve fibers in the adult vertebrate CNS. Glia 2000;29:175-181.

48. Chen MS, Huber AB, van der Haar ME, et al. Nogo-A is a myelinassociated neurite outgrowth inhibitor and an antigen for monoclonal antibody IN-1. Nature 2000;403:434-439.

49. GrandPre T, Nakamura F, Vartanian T, Strittmatter SM. Identification of the Nogo inhibitor of axon regeneration as a Reticulon protein. Nature 2000;403:439-444.

50. Perry VH, Brown MC, Gordon S. The macrophage response to central and peripheral nerve injury. A possible role for macrophages in regen eration. J Exp Med 1987;165:1218-1223.

51. Stoll G, Trapp BD, Griffin JW. Macrophage function during Wallerian degeneration of rat optic nerve: clearance of degenerating myelin and Ia expression. J Neurosci 1989;9:2327-2335.

52. Hering E. Die Lehre vom Binokularen Sehen. Leipzig: Wilhelm Englemann, 1868

53. Wong AMF, Tweed D, Sharpe JA. Adaptations and deficits in the vestibulo-ocular reflex after sixth nerve palsy. Invest Ophthalmol Vis Sci 2002;43:99-111.

54. Wong AM, Tweed D, Sharpe JA. The vestibulo-ocular reflex in fourth nerve palsy: Deficits and adaptation. Vision Res 2002;42:2205-2218.

55. Wong AMF, Sharpe JA. Adaptations and deficits in the vestibulo-ocular reflex after third nerve palsy. Arch Ophthalmol 2002;120:360-368.

56. Zhou W, King WM. Premotor commands encode monocular eye movements. Nature 1998;393:692-695

57. Richards BW, Jones FR, Younge BR. Causes and prognosis in 4,278 cases of paralysis of the oculomotor, trochlear, and abducens cranial nerves. Am J Ophthalmol 1992;113:489-496.

58. King AJ, Stacey E, Stephenson G, Trimble RB. Spontaneous recovery rates for unilateral sixth nerve palsies. Eye 1995;9:476-478. 